\title{
Um Interessante Problema de Vestibular Envolvendo a Não-Conservação da Energia Mecânica em um Referencial Acelerado
}

An interesting academic problem involving the non-conservation of mechanical energy in an accelerated reference system

\author{
Jair C. C. Freitas \\ Departamento de Física, Universidade Federal do Espírito Santo, \\ Vitória - ES, 29060-900, Brasil
}

Recebido em 8 de março, 2002. Aceito em 23 de abril, 2002.

\begin{abstract}
Discutimos detalhadamente a solução de um problema originalmente proposto para um exame vestibular, envolvendo a oscilação de um sistema massa-mola num referencial acelerado e a determinação da amplitude de oscilação a partir do instante em que cessa a aceleração do referencial. Mostramos que o valor dessa amplitude depende essencialmente da relação entre o tempo durante o qual permanece a aceleração do referencial e o período de oscilação. Na situação limite em que o período de oscilação é muito maior do que o tempo de aceleração, o sistema aproxima-se de um sistema conservativo e a amplitude de oscilação pode ser determinada por conservação da energia mecânica. O problema apresentado constitui um interessante exercício para a discussão (em nível médio ou universitário) de temas como forças fictícias, mudanças de coordenadas e não-conservação de energia mecânica em referenciais não-inerciais.
\end{abstract}

The detailed solution of a problem originally proposed for a college-admission exam is discussed. The problem involves the oscillation of a mass-spring system in an initially accelerated referential and the determination of the amplitude of vibration after the referential has reached the rest. We show that the value of this amplitude depends essentially on the relation between the time of acceleration and the period of oscillation. In the limit case when the period of oscillation is much longer than the time of acceleration, the system approaches a conservative system and the answer can be found by applying the principle of conservation of mechanical energy. The presented problem constitutes an interesting exercise for the discussion (at either high-school or college level) of subjects like fictitious forces, coordinates change, and non-conservation of mechanical energy in non-inertial reference systems.

\section{Introdução}

A seguinte questão foi proposta na primeira etapa (aquela prestada por todos os candidatos, independente da área de conhecimento do curso pretendido) do vestibular de 2002 de uma conceituada universidade brasileira: ${ }^{1}$

Um vagão ferroviário move-se em um trecho retilíneo de uma linha ferroviária, com velocidade constante de módulo $\boldsymbol{v}_{o}$. No seu interior, há um bloco de massa $m$ preso à extremidade de uma mola ideal de constante elástica $\boldsymbol{k}$. A outra extremidade da mola está presa ao vagão, conforme a figura abaixo:

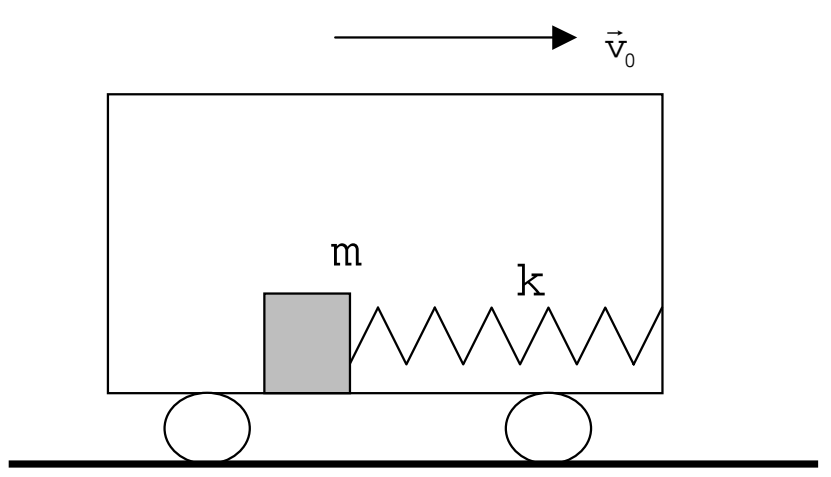

Nesse estado de movimento, a mola está relaxada (não está comprimida nem distendida). A partir de um certo instante, o vagão é freado com aceleração constante $\boldsymbol{a}$, até atingir o repouso. Desprezando-se o atrito do bloco com o piso do vagão, a amplitude de oscilação

\footnotetext{
${ }^{1}$ Omitiremos aqui o nome da universidade, assim como dos cursinhos responsáveis pela correção divulgada nos jornais, para preservar as instituições envolvidas. A questão não foi anulada, a despeito de muitos protestos.
} 
do sistema massa-mola, após o vagão atingir o repouso, é:
A) 0
B) $\sqrt{\frac{k}{m}}$
C) $\frac{m a}{k}$
D) $\sqrt{\frac{m v_{0}^{2}}{k}}$
E) $\frac{m v_{0}}{k}$

O gabarito oficial da universidade indicava como correta a alternativa "C". Após a divulgação do gabarito, a questão foi resolvida de maneira aparentemente simples pelos professores de cursinho, que apresentaram nos jornais a seguinte solução (exatamente como aqui vai escrito, sem nenhuma explicação adicional):

$$
\begin{gathered}
F=F_{e} \\
m a=k x \\
x=\frac{m a}{k}
\end{gathered}
$$

logo: $A=\frac{m a}{k}$

E os comentários apresentados pelos mesmos professores de cursinho nos jornais foram que a referida questão era de nível "normal".

Uma análise mais detalhada do problema revela claramente que a questão, em primeiro lugar, não é de fácil solução, sendo, ao contrário, de um nível bastante elevado para o conteúdo de Física ministrado no ensino médio. Trata-se de um problema que envolve a aplicação da segunda lei de Newton em referenciais acelerados e a obtenção da equação de movimento de um oscilador harmônico submetido a uma força constante.

O aspecto que torna o problema complicado, claro, é o fato de que o bloco não se move em geral com a mesma aceleração do vagão, o que invalida completamente a solução apresentada acima. Embora não tenhamos obtido acesso às estatísticas das respostas objetivas apresentadas pelos alunos, imaginamos que, dentre aqueles alunos que se detiveram em analisar o problema com algum cuidado, grande parte deve ter partido para solucioná-lo aplicando a conservação da energia mecânica ao sistema massa-mola, chegando portanto à alternativa "D". Os alunos menos atentos devem ter marcado a alternativa "B", devido à bem conhecida fórmula para a freqüência de oscilação de um sistema massa-mola típico, sem se aperceberem do problema dimensional com essa alternativa (assim como com a alternativa "E").

Os alunos que "acertaram" a questão, de acordo com o gabarito oficial, devem ter incorretamente raciocinado como se o bloco estivesse sempre em repouso com relação ao vagão. Essa hipótese parece razoável ao nosso senso comum porque estamos acostumados a lidar com sistemas onde existe dissipação de energia e a aceleração varia sempre suavemente de zero até seu valor máximo. Assim, durante a aceleração ou frenagem de um trem (ou automóvel, avião, etc), se observarmos o movimento de um sistema oscilatório, como o sistema massa-mola acima ou um pêndulo simples, perceberemos que o sistema tem sua posição de equilíbrio deslocada mas, após um curto intervalo de tempo, passa a acompanhar solidariamente o movimento do trem. Se a aceleração for retirada subitamente no instante em que o trem atingir o repouso, o sistema oscilará (de forma amortecida) com amplitude inicialmente igual ao deslocamento da sua posição de equilíbrio. Esse raciocínio leva à solução "oficial" da questão de vestibular apresentada (é claro que, com dissipação de energia, a amplitude de oscilação do bloco deve diminuir até zero após a retirada da aceleração, de forma que a alternativa "A" seria a mais apropriada nesse caso). O problema é que nesta questão (i) não há atrito, (ii) a aceleração é aplicada bruscamente e (iii) não há qualquer informação a respeito da relação entre o tempo que o vagão leva para atingir o repouso e o período de oscilação. Assim, não há como supormos que o bloco chegue a se movimentar solidariamente ao vagão. Para que a questão tivesse a solução simples desejada pela equipe que a propôs, seria necessário que o enunciado deixasse claro que o bloco estava em movimento solidário ao vagão durante a frenagem e antes de este atingir o repouso. (Conforme veremos mais adiante, a mesma reposta seria obtida se a questão se referisse somente ao intervalo de tempo durante o qual o vagão é desacelerado, sem entrar na discussão do que acontece após o vagão atingir o repouso.)

O objetivo desta nota é apresentar a solução completa da questão na forma como ela foi proposta, utilizando-se recursos matemáticos certamente acima do nível de ensino médio, mas que permitem um entendimento claro do problema. Veremos que na verdade há várias soluções possíveis, sendo aceitáveis quaisquer das alternativas dimensionalmente corretas ("A", "C" ou "D"). O interesse em discutir em detalhe essa solução não é motivado simplesmente pelo intuito de "corrigir" um problema de vestibular mal formulado, mas também e principalmente pela possibilidade de apresentar um exemplo bastante rico de uma situação que envolve a conservação (ou não) da energia mecânica em referenciais não-inerciais, assunto a respeito do qual percebemos uma certa carência de discussões aprofundadas nos textos clássicos de Física, tanto do nível médio como universitário [1-4]. Após encontrarmos a solução completa do problema, podemos examinar alguns casos particulares e assim ganhar uma compreensão mais profunda a respeito desse aspecto.

Antes de prosseguir, quero ressaltar que em minha opinião o problema, ainda que só tivesse uma alternativa correta, não seria de forma alguma adequado para uma questão de vestibular, principalmente em primeira etapa. A discussão sobre referenciais acelerados é apresentada de forma superficial nos textos clássicos de en- 
sino médio $[1,2]$, e a conservação de energia mecânica nesses sistemas não é tratada com detalhe nem em textos de nível universitário [3,4]. Não é de modo algum justo para com os estudantes de ensino médio propor uma questão que aparentemente tem todos os ingredientes de um sistema conservativo típico (mola ideal, ausência de atrito entre o bloco e o piso), mas onde na verdade ocorre trabalho não conservativo realizado pela força exercida pela mola sobre o bloco. Tenho sérias dúvidas se estudantes de ensino médio teriam a percepção necessária para compreender esse aspecto; por isso a questão apresentada não serve para avaliar o conhecimento dos candidatos ao vestibular. Tal fato fica tanto mais evidente na medida em que os próprios professores de cursinho não se aperceberam da dificuldade do problema e resolveram a questão de forma completamente incorreta, embora coerente com o gabarito oficial.

\section{Solução do problema en- quanto o vagão é desacelerado}

\section{II.1 Referencial inercial}

Consideremos um referencial inercial $S$ em relação ao qual o vagão e o bloco se movem, inicialmente, com velocidade constante de módulo $v_{o}$ ao longo da direção $x$. Tomemos como origem a posição em que o bloco se encontrava em $t=0$, instante em que o vagão começou a ser desacelerado com aceleração constante $a_{x}=-a$. O movimento desacelerado irá durar até o instante $t_{o}=v_{o} / a$. A posição do bloco num instante qualquer entre $t=0$ e $t=t_{o}$ será representada por $x_{B / S}(t)$, o comprimento da mola relaxada será denominado $L$ e o movimento do vagão será descrito pela coordenada $x_{V / S}(t)$ que representa a posição do ponto em que a mola é fixa ao vagão (ver Fig. 1). A elongação $\delta x$ da mola em qualquer instante, que interessa para aplicação da lei de Hooke, será então dada por:

$$
\delta x=x_{B / S}-x_{V / S}+L
$$

(Com essa definição $\delta x$ é negativo para a mola distendida e positivo para a mola comprimida.)

No referencial $S$, a força resultante sobre o bloco é igual à força elástica exercida pela mola, a qual, se assumirmos como válida a lei de Hooke, será dada por:

$$
F_{x}^{(e)}=-k \delta x=k\left(x_{V / S}-x_{B / S}-L\right)
$$

A equação de movimento do vagão entre $t=0 \mathrm{e}$ $t=t_{0}$ será:

$$
x_{V / S}(t)=L+v_{o} t-\frac{1}{2} a t^{2}
$$

Já o movimento do bloco será descrito pela equação diferencial abaixo, obtida após aplicação direta da segunda lei de Newton:

$$
\frac{d^{2} x_{B / S}}{d t^{2}}+\frac{k}{m}\left(x_{B / S}-x_{V / S}+L\right)=0,
$$

com as condições iniciais:

$$
\begin{aligned}
& x_{B / S}(0)=0 \\
& \left.\frac{d x_{B / S}}{d t}\right|_{0}=v_{0}
\end{aligned}
$$

A substituição de (3) em (4) leva à equação diferencial:

$$
\frac{d^{2} x_{B / S}}{d t^{2}}+\frac{k}{m} x_{B / S}=\frac{k}{m}\left(v_{0} t-\frac{a t^{2}}{2}\right)
$$

A solução dessa equação (linear, de segunda ordem e com coeficientes constantes) é encontrada sem maiores dificuldades por meio dos métodos tradicionais de cálculo [5]. Resolve-se inicialmente a equação homogênea, que tem solução harmônica, e a seguir encontra-se uma solução particular da equação inomogênea, a qual é facilmente determinada já que o membro direito da Eq. 7 é um polinômio de segundo grau na variável $t$. Com as condições iniciais (5) e (6), a solução exata da Eq. 7 fica:

$$
x_{B / S}(t)=\frac{m a}{k}(1-\cos \omega t)+v_{0} t-\frac{a t^{2}}{2},
$$

onde $\omega=\sqrt{k / m}$ é a freqüência natural de oscilação do sistema massa-mola. A velocidade do bloco em relação a $S$ é imediatamente obtida por derivação da Eq. 8:

$$
v_{B / S}(t)=\frac{\omega m a}{k} \operatorname{sen} \omega t+v_{0}-a t
$$

Fica claro dessas duas últimas expressões que o movimento do bloco consiste em um movimento harmônico simples (MHS) superposto ao movimento acelerado do vagão (responsável pelos dois últimos termos das Eqs. 8 e 9). Essa descrição fica mais evidente através da análise do problema do ponto de vista do referencial acelerado do vagão, como discutido na seção seguinte.

\section{II.2 Referencial acelerado}

A passagem para o referencial acelerado solidário ao vagão, com origem no ponto em que a mola é fixa ao vagão (ver Fig. 1), é feita de maneira imediata:

$$
x_{B / V}(t)=x_{B / S}(t)-x_{V / S}(t)
$$

Usando (3) e (8):

$$
x_{B / V}(t)=\frac{m a}{k}(1-\cos \omega t)-L
$$

Assim, o movimento do bloco em relação ao vagão é um MHS, com posição de "equilíbrio" deslocada de $m a / k$ para a direita, similar ao movimento de oscilação de um sistema massa-mola fixo numa posição vertical sob ação da força da gravidade [6]. O bloco oscila entre as posições $x_{B / V}=-L$ (que corresponde 
à posição de equilíbrio antes de o vagão ser acelerado) e $x_{B / V}=-L+2 m a / k$. A amplitude do movimento é, portanto, igual a $m a / k$ e a nova posição de "equilíbrio", ou seja, o ponto em que a aceleração do bloco em relação ao vagão é nula, localiza-se em $x_{B / V}=-L+m a / k$. Esta seria exatamente a posição em que o bloco entraria em repouso em relação ao vagão (e conseqüentemente em movimento solidário ao vagão) se houvesse dissipação de energia no sistema.

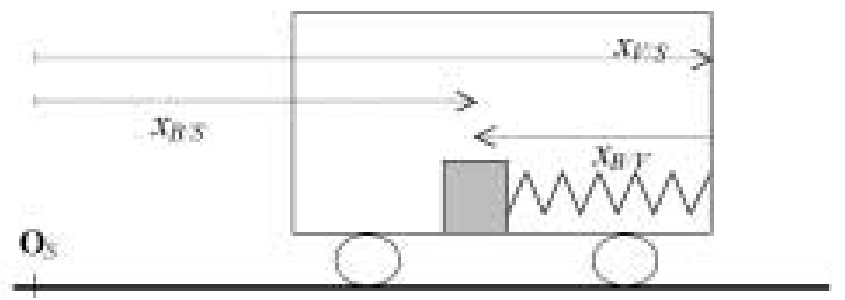

Figura 1. Coordenadas usadas para descrever o movimento do bloco em relação ao referecial $S\left(x_{B / S}\right)$ e em relação ao vagão $\left(x_{B / V}\right) . \mathbf{O}_{S}$ representa a origem do referencial $S$, a qual coincide com a posição do bloco no instante em que o vagão começou a ser desacelerado $(t=0)$.

A solução do problema no referencial do vagão, dada pela Eq. 11, poderia ser obtida de maneira mais direta (e elegante) sem a necessidade de se usar o referencial $S$. Bastaria para tanto empregar o conceito de força fictícia [3,4] no referencial acelerado do vagão. Neste referencial o bloco estaria portanto sujeito à ação de duas forças na direção horizontal: a força elástica dada por (2) e a força fictícia dada por:

$$
F_{x}^{(f)}=-m a_{x}=m a
$$

O movimento do bloco no referencial acelerado é então descrito como um MHS forçado e a solução da equação diferencial que surge ao se empregar a segunda lei de Newton com as forças dadas por (2) e (12), juntamente com as condições iniciais (5) e (6) adaptadas para o referencial do vagão, é exatamente a expressão indicada em (11).

\section{Solução do problema com o vagão em repouso}

Após o vagão atingir o repouso, o movimento do bloco claramente é um MHS com a mesma posição de equilíbrio original (de antes de o vagão começar a ser desacelerado). Podemos achar a amplitude de oscilação do sistema nessa situação considerando que a energia mecânica é conservada a partir do instante em que o vagão deixa de ser acelerado. Basta assim encontrar a energia cinética do bloco e a energia potencial elástica do sistema massa-mola no instante $t=t_{0}=v_{0} / a$, o que pode ser feito tanto no referencial $S$ como no referencial do vagão, já que nesse instante ambos os referenciais são inerciais e estão em repouso relativo. A energia cinética pode ser obtida através de (9):

$$
\frac{m v_{B / S}^{2}\left(t_{0}\right)}{2}=\frac{\omega^{2} m^{3} a^{2}}{2 k^{2}} \operatorname{sen}^{2} \frac{\omega v_{0}}{a}=\frac{m^{2} a^{2}}{2 k} \operatorname{sen}^{2} \frac{\omega v_{0}}{a}
$$

A energia potencial elástica é encontrada a partir da elongação da mola em $t_{o}$, a qual pode ser obtida de (8) ou (11):

$$
\frac{k \delta x^{2}\left(t_{0}\right)}{2}=\frac{m^{2} a^{2}}{2 k}\left(1-\cos \frac{\omega v_{0}}{a}\right)^{2}
$$

Aplicando a conservação da energia mecânica para $t>t_{o}$ :

$$
\frac{m v_{B / S}^{2}\left(t_{0}\right)}{2}+\frac{k \delta x^{2}\left(t_{0}\right)}{2}=\frac{k A^{2}}{2},
$$

onde $A$ é a amplitude desejada. Resolvendo, chegamos a:

$$
A=\frac{m a}{k}\left[2\left(1-\cos \frac{\omega v_{0}}{a}\right)\right]^{1 / 2}
$$

Esta finalmente seria a resposta ao problema proposto. Como era de se esperar, o valor da amplitude depende da relação entre o período do movimento oscilatório $T=2 \pi / \omega$ e o tempo de parada $t_{o}$ do vagão, já que $\omega v_{0} / a=2 \pi t_{0} / T$. Em princípio, qualquer valor desde 0 até $2 m a / k$ é possível, dependendo da posição e da velocidade do bloco no exato instante em que a velocidade do vagão chega a zero e a sua aceleração é subitamente retirada. Fica claro, então, que as respostas "A" e "C" são admissíveis como situações particulares. A amplitude seria igual a 0 (resposta "A"), se no instante $t_{o}$ o bloco estivesse exatamente na posição em que a mola se encontra relaxada (que é a mesma posição em que o bloco estava quando o vagão começou a ser freado, com velocidade nula em relação ao vagão). Já a resposta "C" seria admissível na situação particular em que $\cos \left(\omega v_{0} / a\right)=1 / 2$.

E quanto à alternativa "D"? Ela é dimensionalmente correta, mas haveria uma situação particular em que a amplitude dada por (16) fosse igual a $\sqrt{m v_{0}^{2} / k}$ ? A resposta a essa questão depende fundamentalmente dos valores particulares de $m, a, k$ e $v_{o}$. Consideremos os seguintes valores arbitrários (talvez realistas) para esses parâmetros: $m=1 \mathrm{~kg}, v_{o}=20 \mathrm{~m} / \mathrm{s}, k=10 \mathrm{~N} / \mathrm{cm} \mathrm{e}$ $a=0,2 \mathrm{~m} / \mathrm{s}^{2}$. Obtemos, então, $t_{o}=100 \mathrm{~s}, T=0,2 \mathrm{~s}$, $m a / k=0,2 \mathrm{~mm}$ e $\sqrt{m v_{0}^{2} / k}=0,6 \mathrm{~m}$. É claro que, nessas condições, a resposta "D" estaria muito acima da amplitude correta (em torno de 0,24 mm). Com tais valores numéricos, o bloco efetuaria muitas oscilações completas antes de o vagão atingir o repouso.

A alternativa "D" seria a correta se houvesse conservação da energia mecânica entre $t=0$ e $t=t_{o}$. Embora o bloco não sofra a ação de nenhuma força de atrito, o fato de a força resultante atuando sobre ele depender explicitamente do tempo no referencial inercial $S$ (ver Eqs. 2 e 3) torna o sistema não-conservativo. Em outras palavras, a força exercida pela mola não depende somente da posição do bloco, como é usual em 
sistemas conservativos [3], mas também da posição do ponto em que a mola é fixa ao vagão, sendo este último acelerado. Assim, a energia mecânica não é conservada. Uma forma mais direta para se perceber que a energia mecânica não é conservada numa situação como essa consiste em examinarmos a situação em que o bloco se movimenta solidariamente ao vagão desacelerado. Nesse caso a energia cinética do bloco em relação ao referencial $S$ diminui continuamente, mas a energia potencial elástica armazenada na mola é constante. Fica então claro que a força exercida pela mola não é conservativa quando a própria mola está em movimento acelerado.

Mas existe uma situação limite para o problema original em que nos aproximamos muito de um sistema idealmente conservativo: é quando o tempo de parada do vagão é muito mais curto que o período de oscilação do sistema massa-mola. Nesse caso, praticamente toda a energia cinética que o bloco tinha em relação a $S$ é transferida para a mola na forma de energia potencial elástica. Dito de maneira intuitiva e informal, o bloco não "percebe" a desaceleração do vagão porquanto o tempo de frenagem é muito reduzido. Essa situação poderia ser verificada na prática se o vagão sofresse uma desaceleração muito intensa e/ou se a mola tivesse uma constante elástica muito pequena (uma mola muito comprida, por exemplo [6]). Em qualquer caso, teríamos $t_{o}<<T$. Tomando portanto $\omega v_{o} / a<<1$, podemos expandir a Eq. 16 em série de potências e, retendo o termo de mais baixa ordem na série, obtemos o resultado:

$$
A \cong \frac{m a}{k}\left\{2\left[1-\left(1-\frac{\omega^{2} v_{0}^{2}}{2 a^{2}}\right)\right]\right\}^{1 / 2}=\sqrt{\frac{m v_{0}^{2}}{k}}
$$

Isso mostra que mesmo a alternativa "D", que a princípio parecia ser completamente descabida, é admissível como um caso extremamente particular. Na medida em que o enunciado do problema não estabeleceu restrição alguma sobre os valores numéricos dos parâmetros envolvidos, até essa solução passa a ser possível.

\section{Conclusão}

Conforme havíamos antecipado, qualquer uma dentre as três alternativas dimensionalmente corretas apresentadas na questão de vestibular pode ser admissível, dependendo das relações particulares entre os parâmetros envolvidos no problema. Essa indeterminação, além do grau de dificuldade elevado para o nível do ensino médio, inviabiliza completamente a questão apresentada como uma questão objetiva de vestibular. Por outro lado, o problema mostra-se extremamente rico para uma discussão aprofundada a respeito da não-conservação da energia mecânica em referenciais não-inerciais. Numa discussão em nível universitário, o problema pode ser apresentado como um exercício de grau de dificuldade médio envolvendo conceitos como solução de equações diferenciais nãohomogêneas, forças fictícias, mudanças de referenciais e aproximações por séries de potências. No nível do conteúdo de mecânica do ensino médio, o problema pode ser abordado sem uso das ferramentas matemáticas de cálculo diferencial, desde que seja apresentado o conceito de força fictícia e sejam usados argumentos qualitativos ou semiquantitativos para a obtenção da equação que descreve o movimento do bloco no referencial do vagão (Eq. 11). Quanto à questão da conservação da energia, tanto em nível de ensino médio quanto universitário, a recuperação da situação conservativa no caso limite em que o tempo de parada é pequeno em comparação com o período de oscilação torna o problema reconfortante e permite a aquisição, por parte do aluno, de uma intuição física a respeito das razões que levam o problema a ser em geral nãoconservativo.

\section{Agradecimentos}

Ao Prof. Ricardo C. de Berredo, pelas valiosas discussões iniciais sobre o problema, e aos colegas que leram o manuscrito e apresentaram sugestões enriquecedoras.

\section{Referências}

[1] F. Ramalho, José Ivan C. dos Santos, Nicolau G. Ferraro e Paulo A. de Toledo Soares, Os Fundamentos da Física, Vol. 1, Ed. Moderna, São Paulo, 4a edição (1987).

[2] A. Máximo Ribeiro da Luz e B. Alvarenga Álvares, Física: Volume Único, Ed. Scipione, São Paulo (1997).

[3] D. Halliday, R. Resnick, J. Walker, Fundamentos de Física, Vol. 1, Ed. LTC, Rio de Janeiro, 4a edição (1993).

[4] F. G. Keller, W. E. Gettys e M. J. Skove, Física, Vol. 1, Ed. Makron, São Paulo (1999).

[5] W. E. Boyce e R. C. Diprima, Equações Diferenciais Elementares e Problemas de Valores de Contorno, Ed. Guanabara, Rio de Janeiro, 3a edição (1977).

[6] D. Halliday, R. Resnick, J. Walker, Fundamentos de Física, Vol. 2, Ed. LTC, Rio de Janeiro, 4a edição (1993). 